DOI: https://doi.org/10.11144/Javeriana.umed59-2.mixt

\title{
Malaria mixta: a propósito del diagnóstico de un caso en zona no endémica
}

\section{Mixed Malaria: Regarding the Diagnosis of a Case in a Non-Endemic} Zone

Recepción: 26/10/2016 | Aceptación: 23/11/2017

\author{
Julián E. Barahona-Correa ${ }^{\mathrm{a}}$ \\ Pontificia Universidad Javeriana, Colombia \\ ORCID: http://orcid.org/0000-0002-8244-2958 \\ ANDRÉs JiMÉNEZ \\ Pontificia Universidad Javeriana, Colombia \\ Melissa de Castro \\ Pontificia Universidad Javeriana, Colombia \\ ORCID: http://orcid.org/0000-0003-0605-6868 \\ Juan Carlos López \\ Hospital Universitario San Ignacio, Colombia
}

a Correspondencia: barahonaj@javeriana.edu.co

Cómo citar: Barahona-Correa JE, Jiménez A, De Castro M, López JC. Malaria mixta: a propósito de un caso en zona no endémica. Univ. Med. 2018;59(2). doi: https://doi.org/10.11144/Javeriana.umed59-2.mixt

\section{RESUMEN}

Las infecciones por malaria mixta $(\mathrm{MMx})$ son frecuentemente subdiagnosticadas, a pesar de su alta morbimortalidad. La presencia de más de una especie de Plasmodium spp. y su asociación con síntomas palúdicos configura una $\mathrm{MMx}$. El artículo presenta el caso de una paciente pediátrica diagnosticada con $\mathrm{MMx}$ en un hospital en una zona no endémica. Se hace una breve revisión del tema y su abordaje terapéutico para Colombia.

Palabras clave

malaria; Plasmodium; Plasmodium vivax; Plasmodium falciparum; coinfección.

\section{ABSTRACT}

Mixed-malaria infections are often under recognized, despite their high morbimortality. The presence of more than one species of Plasmodium spp. in association with clinical symptoms configures a mixed-malaria infection. A pediatric patient diagnosed with mixed-malaria infection in a non-endemic zone hospital is described. A brief review and its therapeutic approach for Colombia are presented.

Keywords

malaria; Plasmodium; Plasmodium vivax; Plasmodium falciparum; coinfection.

\section{Introducción}

La malaria o paludismo es la enfermedad parasitaria con mayor impacto en el humano. Cerca de la mitad de la población mundial (aproximadamente 3000 millones de personas) se encuentra expuesta, con más de 210 millones de casos y 600.000 muertes cada año (1). Es causada por el género Plasmodium spp., que cuenta con 5 especies infecciosas 
para el humano (P. falciparum, P. vivax, P. ovale, P. malariae y $P$. knowlesi), y es transmitida por un vector, el mosquito Anopheles (1). En Colombia, más de 12 millones de personas se encuentran expuestas y es endémica en el $75 \%$ del territorio por debajo de los 1500 metros sobre el nivel del mar (m s. n. m), siendo P. vivax y $P$. falciparum las especies más frecuentes (2). Puede presentar síntomas inespecíficos como malestar general, fiebre recurrente (con un patrón particular según la especie) y hepatoesplenomegalia, esta última frecuente en niños. La infección por $P$. falciparum puede generar síntomas neurológicos y multisistémicos $(1,2)$.

Los recientes brotes epidémicos de malaria en Colombia (Cauca, 2014, y Chocó, 2016) $(3,4)$ ilustran su importancia para la salud pública. Factores como el desplazamiento de comunidades, sistemas de vigilancia deficientes, barreras de acceso a pruebas diagnósticas y tratamientos oportunos (a pesar de estar incluidos en el Plan Obligatorio de Salud), al igual que la carencia de personal capacitado, favorecen la expansión de la enfermedad, al limitar las labores de control y eliminación $(2,5)$.

La infección plasmodial mixta (IPMx) es la presencia de formas sexuales o asexuales de dos o más especies simultáneas de Plasmodium spp. (6). La presencia de síntomas palúdicos en el contexto de IPMx se considera malaria mixta (MMx) (6), que presenta una mayor morbimortalidad (3). Su distribución geográfica no es clara; sin embargo, se considera que sigue un patrón coherente con la distribución de las diferentes especies de plasmodios, de tal manera que las coinfecciones solo se presentan entre especies que conviven en un área determinada (6).

En el mundo es incierta la prevalencia de MMx y depende del método diagnóstico utilizado: 2\% por microscopía de luz (p. ej., gota gruesa [GG] o frotis de sangre periférica [FSP]) y hasta el 65\% por reacción en cadena de polimerasa (PCR) (7). En América Latina se calcula una prevalencia del $0,46 \%$ por GG y del $12,8 \%$ por PCR $(6,8)$. Para el 2016, según el Instituto Nacional de Salud, su prevalencia en Colombia superaba el 3\% de los casos reportados (9), donde el departamento del Chocó aportaba cerca del 80\% de los casos, seguido por Amazonas, con el 6\% (8). Su prevalencia en pacientes hospitalizados puede alcanzar hasta el 8\% (10).

La GG y el FSP son sus pilares diagnósticos. La GG presenta una sensibilidad 20 a 30 veces mayor que el FSP; sin embargo, este último permite determinar la especie (2). La PCR presenta el mejor rendimiento diagnóstico (1); pero su disponibilidad y costo limitan su uso generalizado (7). Las pruebas rápidas con dipstick identifican proteínas propias (i. e., lactato deshidrogenasa) del Plasmodium spp. y tienen una sensibilidad del $75 \%$ y una especificidad del $89 \%$ para MMx (7).

En este artículo se presenta el caso de una paciente pediátrica con $\mathrm{MMx}$ diagnosticada en una zona no endémica. Se hace, además, una breve revisión del tema y de su abordaje terapéutico para Colombia.

\section{Descripción del caso}

El caso corresponde a una adolescente de 14 años de edad, natural y residente en Tierra Alta, Córdoba (51 m s. n. m.), procedente de Bogotá (2649 m s. n. m.), donde se encontraba 18 días previos a la valoración en nuestra institución. Consultó al servicio de Urgencias Pediátricas del Hospital Universitario San Ignacio por un cuadro clínico de 5 días de evolución de fiebre no cuantificada con patrón vespertino y escalofríos, emesis, tos, rinorrea, hiporexia y cefalea, esta última de un mes de evolución. Un mes antes había estado hospitalizada por malaria por P. falciparum, diagnosticada con GG con parasitemia de 5680/ $\mu$ l. Recibió tratamiento completo con Arthemeter-Lumefantrine con posterior hemograma normal y GG negativa. Como antecedente de importancia, la paciente había presentado malaria por $P$. vivax a los 9 años, que fue tratada y resuelta. No se conocía el esquema utilizado.

En el examen físico se encontraba afebril, con deshidratación grado II y esplenomegalia grado I. No se evidenció ictericia, síntomas osteomusculares o inyección conjuntival. Se sospechó recrudescencia (i. e., reaparición de 
parásitos en sangre, relacionada con resistencia a fármacos) versus recaída (i.e., reactivación de hipnozoítos) de paludismo.

Se obtuvo una tomografía axial computarizada simple de cráneo y radiografía de tórax, ambas normales; así como las pruebas de función renal, glucemia y transaminasas. La inmunoglobulina (IgM) para dengue fue negativa. Se evidenció trombocitopenia $(98.700 / \mu \mathrm{l})$ sin alteración en otras líneas celulares e hiperbilirrubinemia indirecta $(1,65 \mathrm{mg} / \mathrm{dl})$. La ecografía abdominal confirmó esplenomegalia $(135 \mathrm{~mm})$. Se realizó una GG que identificó Plasmodium spp. (9840 parásitos/ $\mu$ l) y un FSP que reportó $P$. vivax. Se diagnosticó recaída compatible con MMx. Se inició tratamiento con cloroquina y primaquina, con posterior curación. La paciente egresó y continuó con controles ambulatorios con seguimiento paraclínico, sin alteración alguna.

\section{Discusión}

\section{Resumen y enfoque}

La fiebre asociada a cefalea, síntomas gripales y malestar general pueden presentarse en numerosas enfermedades tropicales, entre las que destaca la malaria, dada su alta morbimortalidad (2). El enfoque clínico del síndrome febril agudo en Colombia ha sido revisado recientemente por Cortés y colaboradores (11).

Usualmente, se considera que la reaparición de fiebre tras un tratamiento antimalárico completo debe considerarse falla terapéutica. Sin embargo, nuestro caso ilustra la importancia de realizar una nueva prueba de sangre en busca de Plasmodium spp., como ha sido reportado previamente (12).

El dilema médico se centraba en una recrudescencia del $P$. falciparum frente a una recaída compatible con MMx. La infección previa tratada por P. falciparum asociada a la aparición de síntomas tras 2 semanas en una zona sin riesgo de transmisión y un FSP positivo para P. vivax diagnosticaban MMx (P. falciparum-P. vivax). Dado que el tratamiento para $P$. falciparum no contiene un esquizonticida tisular, la curación radical de MMx no es posible y permite la recaída (i. e., reactivación de hipnozoítos, que son partículas parasitarias que permanecen latentes en el tejido hepático), ya que este esquema cubre únicamente formas circulantes de P. vivax $(1,2)$.

\section{Malaria mixta}

La mayoría de casos de malaria son producidos por una única especie (1). Sin embargo, la IPMx/ $\mathrm{MMx}$ es subdiagnosticada y presenta mayor morbimortalidad $(7,8)$. Siempre que diferentes especies de Plasmodium spp. coexistan en una misma área, la posibilidad de una IPMx es alta (7). Así mismo, la IPMx es común en el vector (Anopheles) (6).

Las IPMX/MMx suelen detectarse después de la reactivación de una especie de Plasmodium spp. no identificada al inicio del tratamiento de un paciente con malaria (7). P. vivax y $P$. ovale pueden dar recaídas, por su capacidad de generar hipnozoítos, que pueden reactivarse en numerosos escenarios (13). El tiempo de reactivación depende del fármaco utilizado: menor a 4 semanas, con antimaláricos de vida media corta (i. e., derivados de artemisina o quinina), y entre 4 y 6 semanas, con compuestos de vida media larga (i. e., mefloquina o cloroquina) (7). En Tailandia, hasta el 30\% de los pacientes con malaria por P. falciparum desarrollaron parasitemia por $\mathrm{P}$. vivax sin reexposición, hallazgo coherente con reactivación de hipnozoítos (14).

Sin embargo, P. falciparum puede sufrir un proceso de "reactivación" similar. Looareesuwan y colaboradores (14) reportaron que hasta un $10 \%$ de los pacientes con malaria por P. vivax desarrollaron parasitemia por P. falciparum sin reexposición en menos de 2 semanas de inicio del tratamiento. Este fenómeno puede deberse a formas asexuadas eritrocíticas que no responden al tratamiento para P. vivax (como la cloroquina). No se descartan errores en el momento del diagnóstico o una posible prolongación de la fase intrahepática de P. falciparum durante infecciones mixtas (7).

La interacción entre especies coinfectantes parece modular la severidad de la IPMx/MMx 
(7). Este hecho puede estar relacionado con que P. falciparum infecta todos los eritrocitos y reduce el número de células jóvenes disponibles para la infección por $P$. vivax. También se ha planteado que la severidad del cuadro por $P$. falciparum puede disminuir, dada una menor parasitemia por $P$. vivax para generar fiebre, lo que permite un tratamiento precoz (7). La identificación tardía de coinfección con $P$. falciparum predispone a cuadros más severos por un tratamiento inapropiado $(1,7)$.

No es claro si existe un cuadro clínico propio de la MMx. Se han reportado mayor frecuencia de fiebre mayor a 38 grados en $\mathrm{MMx}(88 \%$ vs. $60 \%$ y $47 \%$ por $P$. falciparum y por $P$. vivax, respectivamente) (15), trombocitopenia (16) y anemia severa, probablemente secundaria al volumen de parásitos (17). El abordaje terapéutico presenta evidencia limitada: al sospechar un cuadro de IPMx/MMx, se debe iniciar tratamiento para $P$. falciparum, ajustado a los perfiles de resistencia locales, dada su alta morbimortalidad. Siempre que haya presencia de $P$. vivax o $P$. ovale, un agente hipnozonticida debe ser adicionado. La tabla 1 resume el esquema terapéutico para Colombia, que se encuentra en la Guía de Atención Clínica de Malaria (2).

Tabla 1. Tratamiento de malaria no complicada por la asociación de P. falciparum y P. vivax

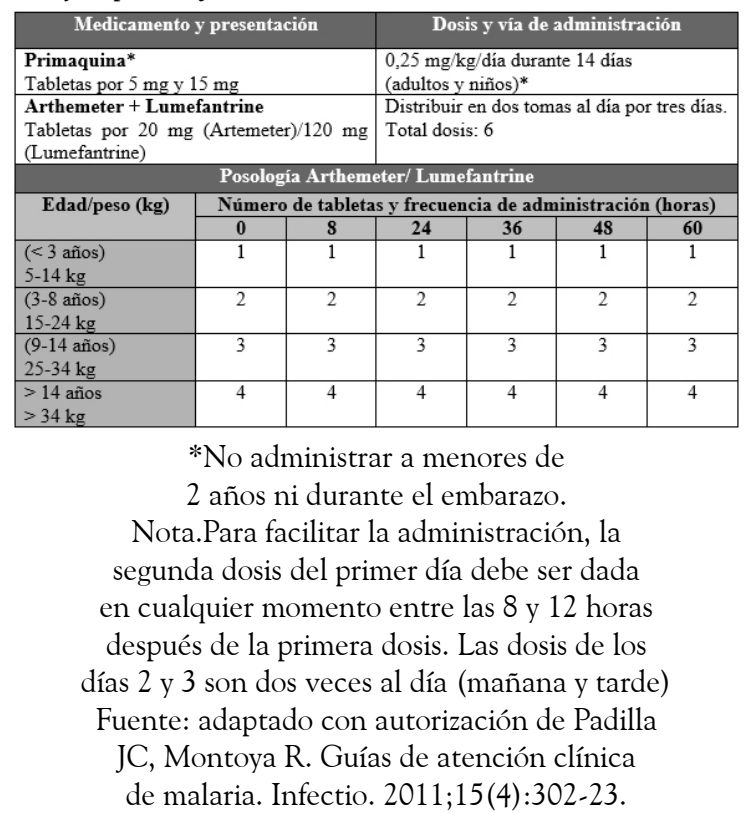

\section{Conclusión}

Nuestro caso ilustra la importancia del diagnóstico y el tratamiento temprano de la MMx. El enfoque sindromático de los síntomas inespecíficos en un paciente con antecedente epidemiológico debe ser la piedra angular del abordaje, como ha sido revisado en población pediátrica (18). Adicionalmente, se resalta la importancia de fortalecer el acercamiento a la medicina tropical en las escuelas de medicina en zonas no endémicas, particularmente en un país con alta prevalencia de enfermedades tropicales como Colombia.

\section{Referencias}

1. White NJ, Pukrittayakamee S, Hien TT, Faiz MA, Mokuolu OA, Dondorp AM. Malaria. Lancet. 2014;383(9918):723-35.

2. Padilla JC, Montoya R. Guías de atención clínica de malaria. Infectio. 2011;15(4):302-23.

3. Borbón M, Campo A, Restrepo C. Brote de malaria en los municipios de Guapi y Timbiquí, Cauca, Colombia, 2014. Inf Quinc Epidemiológico Nac IQEN. 2014;19(15).

4. Ministerio de Salud y Protección Social de Colombia. Minsalud atendió brote de malaria en Chocó. Boletín de Prensa 089. 2016.

5. Blair S. [Challenges for the elimination of malaria in Colombia: a problem of knowledge or of power]. Biomédica. 2012;32 Suppl 1:31-148.

6. Carmona-Fonseca J, Arango E. Malaria mixta: revisión general. Médicas UIS. 2012;25(1):45-54.

7. Mayxay M, Pukrittayakamee S, Newton PN, White NJ. Mixed-species malaria infections in humans. Trends Parasitol. 2004;20(5):233-40.

8. Carmona-Fonseca J, Arango-Flórez E. Malaria mixta: prevalencia en Colombia y América Latina. Iatreia. 2012;25(4):334-46. 
9. Instituto Nacional de Salud. Malaria. Boletín Epidemiológico Semanal. Semana 52.2016.

10. González L, Guzmán M, Carmona-Fonseca J, Lopera T, Blair S. Características clínico-epidemiológicas de 291 pacientes hospitalizados por malaria en Medellín (Colombia). Acta Médica Colomb. 2000;25:163-70.

11. Cortés JA, Romero-Moreno LF, AguirreLeón CA, Pinzón-Lozano L, Cuervo SI. Enfoque clínico del síndrome febril agudo en Colombia. Infectio. febrero de 2016:39-50.

12. Smith A, Denholm J, Shortt J, Spelman D. Plasmodium species co-infection as a cause of treatment failure. Travel Med Infect Dis. 2011;9(6):306-9.

13. Nadjm B, Behrens RH. Malaria: an update for physicians. Infect Dis Clin North Am. 2012;26(2):243-59.

14. Looareesuwan S, White NJ, Chittamas S, Bunnag D, Harinasuta T. High rate of Plasmodium vivax relapse following treatment of falciparum malaria in Thailand. Lancet. 1987;2 (8567):1052-5.

15. McKenzie FE, Smith DL, O'Meara WP, Forney JR, Magill AJ, Permpanich $\mathrm{B}$, et al. Fever in patients with mixed-species malaria. Clin Infect Dis. 2006;42(12):1713-8.

16. Beg MA, Ali SS, Haqqee R, Khan MA, Qasim Z, Hussain R, et al. Rapid immunochromatography-based detection of mixed-species malaria infection in Pakistan. Southeast Asian J Trop Med Public Health. 2005;36(3):562-4.

17. Genton B, D'Acremont V, Rare L, Baea K, Reeder JC, Alpers MP, et al. Plasmodium vivax and mixed infections are associated with severe malaria in children: a prospective cohort study from Papua New Guinea. PLoS Med. 2008;5(6):e127.

18. Flores MS, Hickey PW, Fields JH, Ottolini MG. A "syndromic" approach for diagnosing and managing travelrelated infectious diseases in children. Curr Probl Pediatr Adolesc Health Care. 2015;45(8):231-43. 